Pecvnia, Monográfico (2008), pp. 19-31

\title{
Marco conceptual para el análisis de la oferta sanitaria
}

\author{
Juan Ventura Victoria \\ ventura@uniovi.es \\ Universidad de Oviedo \\ Dpto. de Administración de Empresas \\ Avda. de El Cristo, s/n \\ 33071 Oviedo (España)
}

El artículo analiza la organización de la atención sanitaria pública en España, mediante un marco conceptual que permite integrar los aspectos más relevantes de la misma. Asimismo, se profundiza en los aspectos organizativos de los hospitales descritos como Burocracias Profesionales, Meritocracias y Organizaciones Intensivas en Conocimientos, aspectos que condicionan la gestión de los mismos. Finalmente, mediante las aportaciones de la teoría de los Recursos y Capacidades se concluye que la gestión sanitaria implica la gestión del conocimiento que reside en el capital humano altamente especializado, el capital organizativo acumulado y la reputación conseguida con el paso del tiempo, todo ello en un entorno tecnológico de alta complejidad y elevado dinamismo.
How public health is organized in Spain is analyzed through using a theoretical framework which includes the most relevant issues regarding this topic. Likewise, this paper deals with organizational aspects from hospitals which determine the way they are managed, such as Professional Bureaucracies, Meritocracies, and Knowledge-Intensive Organizations. Finally, drawing on Resource-Based View contributions, this article concludes that health management, which occurs in a technological, highly complex and dynamic environment, involves the management of knowledge derived from three sources: highly specialized human capital, accumulated organizational capital, and reputation. 


\section{INTRODUCCIÓN}

Desde un punto de vista que facilite el análisis económico Grossman (1972) considera la salud "como un bien de capital, un stock del que se deriva un flujo de días vividos saludablemente y que para ser mantenido requiere efectuar inversiones". La cuestión estriba en conocer cuales son las inversiones que afectan positivamente a la salud, y la respuesta dista de estar clara, al ser la salud la resultante de múltiples factores, entre los que se pueden destacar la genética, el medio ambiente (social y económico), los hábitos y estilos de vida y la atención sanitaria. El objetivo de este capítulo consiste en describir la oferta sanitaria, entendiendo por tal el conjunto de medios asistenciales disponibles para prestar atención sanitaria a una población determinada. Para ello se estudiará el conjunto de recursos y capacidades que conforman el complejo entramado de un sistema sanitaria moderno y las características más relevantes del mismo.

\section{SANITARIA}

\section{MARCO CONCEPTUAL PARA EL ANÁLISIS DE LA ATENCIÓN}

El sistema sanitario público en España se puede considerar que responde al modelo denominado Servicio Nacional de Salud, caracterizado por la financiación pública mediante impuestos, universalización de la atención sanitaria, provisión y gestión predominantemente públicas. El modelo sanitario se ha completado recientemente con el traspaso de competencias sanitarias a todas las Comunidades Autónomas, lo que introduce cierta variedad en el funcionamiento y resultados, siendo este uno de los problemas pendientes de cara al futuro. El funcionamiento del sistema sanitario se puede modelizar tomando como punto de partida las necesidades y demandas de la población objetivo. Las necesidades de atención sanitaria son evaluadas de acuerdo a criterios técnicos a partir de estudios epidemiológicos y establecimiento de premisas sobre el estado de la ciencia médica y la tecnología disponible. En cambio la demanda de atención sanitaria expresa la elección del consumidor al comparar los beneficios y costes en que incurre para obtener un servicio determinado o para garantizar una determinada cobertura. Si bien ambos conceptos están estrechamente relacionados no siempre son coincidentes, pudiendo haber necesidad de 
atención sanitaria sin que se demande la misma o bien demanda sin necesidad. En el ámbito sanitario la evaluación de las necesidades y la satisfacción de las demandas están muy vinculadas al criterio de la profesión médica y al progreso de la medicina, lo cual conlleva que la oferta asistencial influye y condiciona la demanda.

Para satisfacer las demandas de atención sanitaria de la población se requiere definir mediante qué mecanismos se van a regular las transacciones (intercambios) entre los demandantes y los oferentes de servicios sanitarios. El mercado y la planificación son las dos formas puras de asignación de recursos. En un sistema de mercado los precios transmiten la información y los incentivos adecuados y la interacción entre la oferta y la demanda determina qué servicios se prestarán y quién los obtendrá. La segunda alternativa es la planificación, en este caso el gobierno mediante las autoridades sanitarias establece la oferta asistencial y regula el proceso de acceso a la misma.

En un sistema predominantemente público como el nuestro la planificación es la forma dominante, jugando el mercado un papel residual para aquellas prestaciones no cubiertas por el sistema público (por ejemplo determinadas prestaciones buco dentales) o bien para sustituir o complementar la oferta pública con servicios privados (contratación de seguros privados, lo que conduce a una doble cobertura para casi un $20 \%$ de la población española). La oferta asistencial total estará integrada por el conjunto de medios asistenciales (humanos, materiales, técnicos y organizativos) de titularidad pública (o privada) al servicio del sistema público y por la oferta privada.

A partir de los medios asistenciales dotados se genera un flujo de actividad asistencial cuantificable, como son estancias, intervenciones, consultas, pruebas diagnósticas, etc. con la finalidad de mejorar la salud de la población atendida. Si se compara la dotación de medios asistenciales con medidas que recojan el uso que de los mismos se ha hecho se puede considerar la eficiencia. En un sentido restringido la eficiencia en la gestión se mide por la obtención de un producto o servicio minimizando los costes necesarios para su obtención; comparando los recursos asignados con la cantidad de servicios prestados podemos inferir la eficiencia productiva y de gestión de diferentes instituciones sanitarias y efectuar comparaciones entre ellas.

Finalmente, las actividades sanitarias se justifican por el impacto positivo que puedan ejercer sobre la salud, si bien dicho impacto 
resulta en muchas ocasiones difícil de conocer con exactitud. Dado el estado actual de la ciencia médica, podemos definir la eficacia de un determinado proceso asistencial como la probabilidad de que un individuo, en una población definida se beneficie de la aplicación de una tecnología médica a la resolución de un problema de salud determinado. Dicha eficacia se establece habitualmente de forma experimental y con validez universal. En cambio la efectividad también pretende medir la probabilidad de que un individuo de una población definida, se beneficie de la aplicación de un tratamiento, pero en las condiciones reales de aplicación por el equipo asistencial médico (Ortún 1990). En consecuencia la eficacia marca el límite superior de beneficios en la actividad asistencial, mientras que la efectividad traduce en la práctica real la forma en que se utilizan los conocimientos médicos. La calidad de la atención sanitaria se puede definir como la diferencia entre la eficacia y la efectividad alcanzada dados los recursos empleados.

Figura 1: Marco conceptual para el análisis de la atención sanitaria

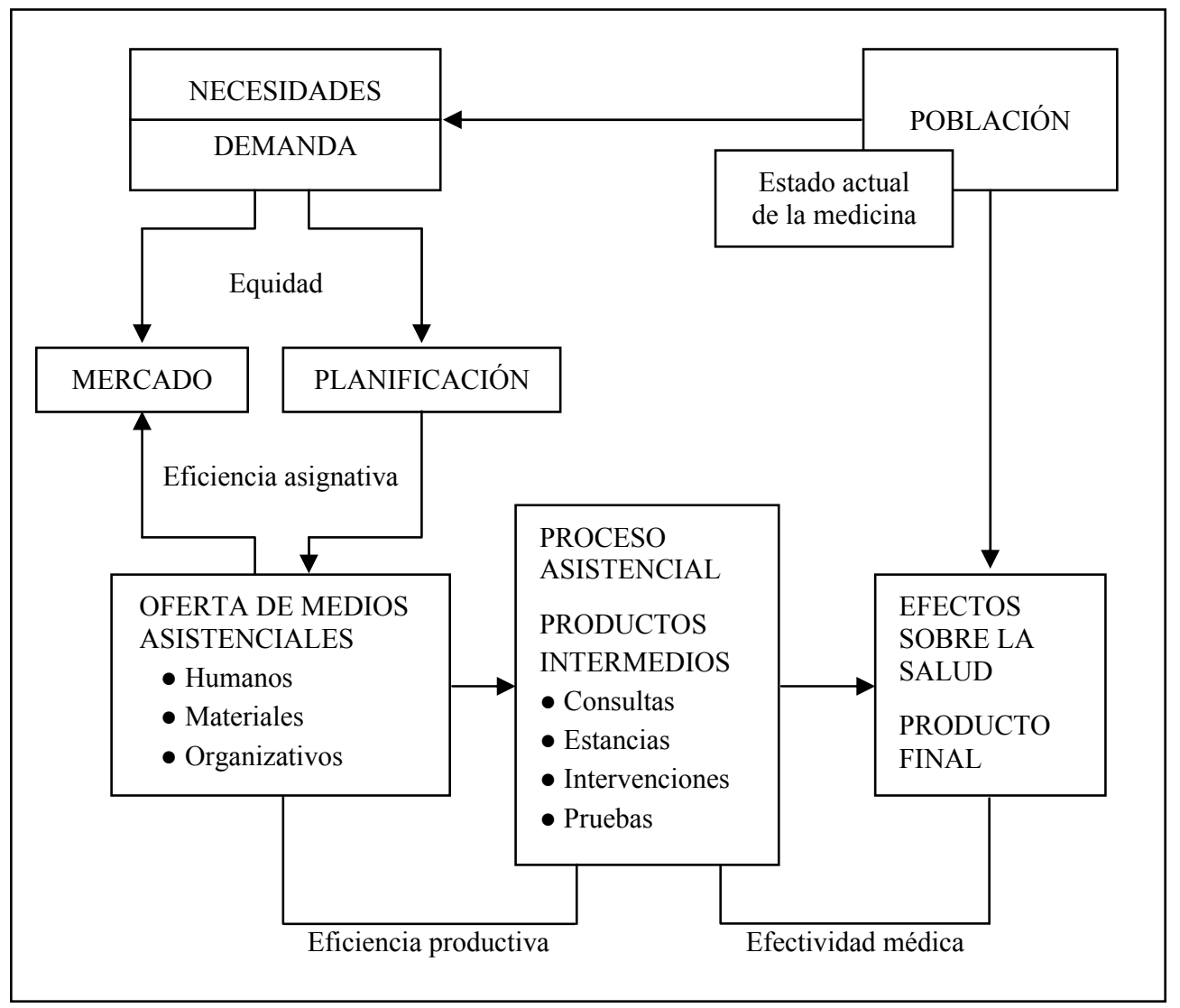


En la Figura 1 se integran todos estos aspectos que permiten plantear un marco conceptual para el análisis de la atención sanitaria. En primer lugar se ha de determinar desde una perspectiva política la forma en que se van a gobernar las diferentes transacciones entre la demanda de atención sanitaria y la oferta asistencial. Como hemos señalado la planificación de medios asistenciales por parte de las autoridades sanitarias es la forma dominante en nuestro sistema sanitario. La forma en que dicha planificación se efectúa va a condicionar el grado de equidad alcanzado en la distribución y uso de los recursos entre la población, así como en el grado de eficiencia asignativa. En el corto plazo la oferta asistencial estará fijada y la variable relevante pasa a ser el grado de eficiencia alcanzado en la gestión por las diferentes instituciones sanitarias que prestan sus servicios. Finalmente se ha de conocer el grado de efectividad médica, o dicho en otros términos la calidad de los servicios prestados.

La interrelación de todos estos aspectos determina finalmente la cantidad, calidad, coste y distribución de los servicios sanitarios obtenidos a partir de unos recursos y conocimientos dados. La resultante final se mide por el efecto diferencial del sistema sanitario sobre la salud de la población.

\section{ANÁLISIS DE LA OFERTA SANITARIA}

\section{Introducción a la oferta asistencial}

Si bien el proceso de transferencias a las Comunidades Autónomas introduce cierta variedad en las características de la oferta sanitaria a grandes rasgos cabe señalar aspectos comunes a la misma. En España la existencia de un seguro obligatorio se ha combinado con una red de centros asistenciales de titularidad pública, tanto en atención primaria como especializada, red que se completa de forma subsidiaria con la participación de centros concertados. En este modelo la financiación pública obligatoria mediante impuestos elimina los riesgos de la selección adversa y logra contener los gastos a nivel macroeconómico, pero a cambio surgen los problemas inherentes a la falta de incentivos y a una clara delimitación de responsabilidades.

Las autoridades sanitarias a partir de la financiación establecida deberán priorizar los objetivos a alcanzar, asignar los recursos 
mediante la utilización de la autoridad o jerarquía, trasmitir la información hacia los destinatarios de la misma, coordinar mediante una estructura organizativa el logro de objetivos comunes a partir de una amplia división del trabajo, y a medida que la complejidad de la organización aumente, se deberá descentralizar parte de la capacidad decisoria. Esta cesión de poder conlleva la necesidad de supervisar y motivar a los diferentes participantes a fin de hacer compatibles los objetivos individuales con los de la organización en su conjunto.

La utilización de sistemas de asignación de recursos determinados centralmente, por medio de autoridades públicas exige disponer de enormes cantidades de información tanto en el establecimiento de los planes (qué se pretende, para quién) como en el control de los resultados obtenidos. Además en este proceso de delegación de poder para tomar decisiones pueden darse desviaciones en el cumplimiento de los objetivos. Serra Ramoneda (1993) sintetiza la cuestión con gran precisión cuando compara la relación de agencia que se establece entre una empresa privada frente a una pública:

En el caso de la empresa pública, esta relación de agencia es más compleja porque está formada por tres niveles. En la base como propietarios, están los ciudadanos, pues sobre ellos recaerá la renta residual que se consiga. Cuando esta sea negativa, se verán obligados a pagar más impuestos para hacer frente al déficit y garantizar la continuidad de la empresa. Por el contrario si el resultado es positivo, el presupuesto público estará más desahogado y la presión fiscal no será tan intensa. A la vez estos propietarios, son en mayor o menor medida usuarios de los bienes o servicios que la empresa produce, por lo que tienen interés en que estos sean abundantes y se les ofrezca al menor precio posible. Estos propietarios nombran mediante el oportuno trámite político, a unos representantes a los que delegan la responsabilidad de definir la política de la empresa. Al final de cada período electoral expresarán con su voto su juicio sobre su comportamiento. Estas instancias políticas, a su vez, designan, orienta y supervisan a los funcionarios a quienes se encarga la gestión de la empresa.

En el segundo escalón de la relación de agencia las autoridades políticas delegan en los gestores o administradores para que estos alcancen los objetivos señalados. $Y$, este es uno de los problemas más cruciales a la hora de juzgar la eficiencia de este mecanismo de asignación de recursos, la falta de claridad en los objetivos a alcanzar, 
debido a que estos se expresan a menudo de forma vaga e imprecisa cuando no de forma contradictoria.

Finalmente, la ausencia de mecanismos de control externo como el suministrado por la cotización bursátil en las grandes sociedades anónimas, o la competencia en el mercado de servicios, hacen posible que los gestores puedan desviarse de forma sensible del curso de acciones necesario para alcanzar la máxima eficiencia en el empleo de los recursos públicos asignados, lo que genera una laxitud organizativa o ineficiencia $x$.

\section{Análisis de la atención primaria / atención especializada}

Una vez hechas estas consideraciones que enmarcan las dificultades inherentes a la gestión pública de la sanidad, se analiza de forma más concreta las características organizativas de la oferta de medios asistenciales en el sistema sanitario público español.

El dispositivo sanitario asistencial público se configura idealmente como una red de centros asistenciales ordenados territorial y jerárquicamente para prestar atención sanitaria en función de la gravedad de la patología. A tal fin se establece la separación entre la atención primaria y la atención especializada. La atención primaria, además de atender la patología menos compleja y tener a su cargo labores fundamentales en el ámbito de la prevención sanitaria y la educación, cumple otra función esencial, al servir de filtro de entrada a los hospitales, canalizando los pacientes que requieren diagnósticos $\mathrm{y} / \mathrm{o}$ tratamientos especializados que sólo estos centros pueden ofrecer.

La oferta asistencial en el ámbito de la Atención Primaria ha sido reformada profundamente con la introducción de los Centros de Salud y los Equipos de Atención Primaria. Estos equipos están integrados por médicos y otros especialistas que trabajan en jornada completa y son retribuidos con un salario al que se le agrega un pago capitativo de menor cuantía. La percepción del nuevo sistema es que el mismo ha mejorado el uso de historias clínicas, protocolos de actuación, se han reducido las prescripciones, se ha aumentado la capacidad resolutiva (lo que ha permitido reducir las derivaciones) y se ha aumentado el tiempo medio de consulta, todo lo cual implica una mejor calidad del servicio prestado. Si bien son muchos los problemas que aún persisten, y prueba de ello el notable aumento de los ingresos por urgencia en los centros hospitalarios. 
A su vez dentro de la atención especializada se establece una ordenación en función de medios técnicos y humanos, de tal forma que determinados hospitales se constituyen en centros de referencia para patologías altamente complejas y otros hospitales actúan a nivel comarcal atendiendo a patologías más sencillas y derivando hacia centros con más recursos las patologías que no pueden atender.

Por tanto el dispositivo asistencial necesario para atender las necesidades y demandas de la población se puede considerar como una red donde se establecen relaciones de interdependencia jerarquizadas, actuando a modo de sistema, en tanto en cuanto el funcionamiento de cada parte afecta al funcionamiento del conjunto.

La plasmación real de este planteamiento dista de haberse conseguido, de hecho desde el punto de vista organizativo el sistema sanitario adolece de la falta de continuidad entre la Atención Primaria y la Atención Especializada. En la práctica existe un claro fraccionamiento o división entre ambos estamentos, lo que ocasiona problemas de accesibilidad, duplicación de pruebas, y falta de diálogo para establecer protocolos de actuación que aseguren la división del trabajo óptima. Como señalan Ortún y López Casanovas (2002):

La integración, real o virtual, puede mitigar la actual falta de continuidad entre $A P$ y $A E$, pero, en última instancia, el que cada problema sea tratado en el lugar más adecuado -el más resolutivo- y que no se retengan casos ni se deriven innecesariamente constituye un problema clínico. No habrá buena medicina sin continuidad y eficiencia en la atención sanitaria, sin objetivos comunes, sin conocimiento mutuo AP/AE y sin el establecimiento conjunto de pautas de actuación.

\section{El hospital como organización: La burocracia profesional}

El hospital se puede definir como una institución que dispone de medios humanos, técnicos y organizativos para prestar atención sanitaria de forma continuada a patologías complejas. Desde el punto de vista de la organización de la actividad asistencial el hospital se puede entender como una empresa que trabaja bajo pedido; cada paciente puede ser considerado como un proyecto que requiere la aplicación de diferentes tecnologías y trabajos; en consecuencia el hospital presenta una estructura tipo taller, esto es agrupa los medios técnicos y humanos de que dispone en áreas funcionales especializadas. De esta forma cada paciente pasará 
por cada estación de trabajo en función de las necesidades específicas que requiera.

En toda organización con una cierta división del trabajo se precisa una estructura organizativa que permita la coordinación de las diferentes actividades. Mintzberg, en su ya clásico trabajo, La estructuración de las organizaciones, considera que existen cinco mecanismos básicos de coordinar las actividades: adaptación mutua, supervisión directa, normalización de procesos, normalización de resultados y normalización de habilidades. El hospital se caracteriza por utilizar el último de los mecanismos, la normalización de las habilidades; mediante este mecanismo se especifica el tipo de preparación requerida para la realización del trabajo, normalización que se complementa con un período de aprendizaje práctico y adoctrinamiento.

La descripción del hospital como una burocracia profesional, se debe a que su trabajo es predecible, pero a diferencia de las burocracias maquinales donde se puede normalizar los procesos de trabajo y supervisar los mismos, los hospitales contratan a profesionales para su núcleo de operaciones, confiriéndoles a continuación un amplio grado de control sobre su propio trabajo. De esta forma el médico (o si se prefiere el servicio médico) dispone de una amplia autonomía y capacidad de decisión sobre la forma de trabajo, dada la dificultad de utilizar adecuadamente el resto de los mecanismos de coordinación señalados.

Profundizando algo más en las características de los hospitales, se pueden diferenciar tres tipos de procesos o actividades productivas que se desarrollan en diferente medida (García y Ventura 1997). Los procesos tipo I agrupan todas aquellas actividades rutinarias (asistenciales o de apoyo) de las que se puede afirmar con certeza la eficacia de las mismas. Los procesos tipo II en cambio, reflejan aquellas actividades de naturaleza compleja, protocolizada en cierto grado, pero no exentas de incertidumbre relativa a la identificación precisa de las situaciones que exigen su aplicación, si bien dicha incertidumbre se encasilla en contingencias para las cuales el profesional sanitario dispone de un conjunto de programas normativos para el diagnóstico y el tratamiento. Por último los procesos tipo III tienen como primera misión configurare a sí mismos como procesos eficaces, dado que existen demandas de atención sanitaria para las que no se conocen procesos cuya eficacia haya sido probada empíricamente. 
Cada tipo de proceso conlleva unas exigencias diferentes. Así la búsqueda de la eficiencia en el uso de los recursos asignados debe ser el elemento prioritario para los procesos cuya eficacia está probada y recíprocamente lograr la eficacia en el diagnóstico y el tratamiento pasa a ser prioritario cuando se desconoce la forma de adecuada de actuar (nuevas patologías, nuevos tratamientos para patologías conocidas). $\mathrm{Si}$ bien conviene destacar que la gran mayoría de la actividad asistencial prestada se corresponde con los procesos tipo II, que requieren una adecuada combinación de medidas tendentes a asegurar la máxima efectividad posible y la eficiencia en el uso de los recursos asignados, equilibrio que dista de ser fácil de lograr (Cuadro 1 ).

Cuadro 1: Tipos de procesos y estructuras organizativas

\begin{tabular}{|l|c|l|l|l|l|}
\hline $\begin{array}{l}\text { Estructuras } \\
\text { organizativas }\end{array}$ & $\begin{array}{l}\text { Tipo de } \\
\text { proceso }\end{array}$ & $\begin{array}{l}\text { Instrumento } \\
\text { coordinador }\end{array}$ & Naturaleza & $\begin{array}{l}\text { Mecanismo } \\
\text { de control }\end{array}$ & $\begin{array}{l}\text { Condiciones ideales } \\
\text { de funcionamiento }\end{array}$ \\
\hline $\begin{array}{l}\text { Burocracia } \\
\text { Maquinal }\end{array}$ & I & $\begin{array}{l}\text { Normalización } \\
\text { de procesos de } \\
\text { trabajo }\end{array}$ & $\begin{array}{l}\text { Medios y fines } \\
\text { especificados en } \\
\text { detalle }\end{array}$ & $\begin{array}{l}\text { Jerárquico y } \\
\text { normalizado }\end{array}$ & $\begin{array}{l}\text { Tareas sencillas y } \\
\text { previsibles }\end{array}$ \\
\hline $\begin{array}{l}\text { Burocracia } \\
\text { Profesional }\end{array}$ & II & $\begin{array}{l}\text { Normalización } \\
\text { de habilidades }\end{array}$ & $\begin{array}{l}\text { Fines especificados y } \\
\text { medios alternativos }\end{array}$ & $\begin{array}{l}\text { Negociación } \\
\text { Dirección por } \\
\text { objetivos (DPO) }\end{array}$ & $\begin{array}{l}\text { Tareas complejas pero } \\
\text { protocolizadas. } \\
\text { Ej. Tratamiento de } \\
\text { patologías convencionales }\end{array}$ \\
\hline Adhocracia & III & $\begin{array}{l}\text { Adaptación } \\
\text { mutua }\end{array}$ & $\begin{array}{l}\text { Fines generales } \\
\text { especificados y medios } \\
\text { sin determinar }\end{array}$ & $\begin{array}{l}\text { Mecanismos de } \\
\text { coordinación } \\
\text { flexibles }\end{array}$ & $\begin{array}{l}\text { Tareas complejas y } \\
\text { novedosas (no previsibles) } \\
\text { Nuevas patologías }\end{array}$ \\
\hline
\end{tabular}

Las burocracias profesionales se corresponden en términos de poder con una meritocracia, caracterizada por que el poder se desliza hacia los profesionales que controlan el recurso más valioso y básico para el funcionamiento de la organización, esto es el conocimiento especializado. Además, en la medida que el resultado final de la actividad organizativa no sea la consecuencia de interdependencias secuenciales o recíprocas entre diversos departamentos, sino más bien la suma de las actividades de los diferentes departamentos o unidades que la componen, cada unidad funcional (departamento o servicio) buscará la optimización de sus recursos frente a los de los demás, lo cual desata los comportamientos políticos o actividades de influencia (Ejercer influencias y presiones para lograr una mayor asignación de recursos, sobre todo en ausencia de medidas precisas del rendimiento profesional).

La situación descrita en términos de poder como meritocracia se ha ido modificando paulatinamente debido a que las autoridades 
sanitarias responsables del cumplimiento de objetivos globales del sistema sanitario han ido introduciendo medidas tendentes a controlar el crecimiento del gasto sanitario y la calidad de los servicios prestados. Ello ha generado una alteración del status quo previo en tanto en cuanto al poder de los profesionales (meritocracia) se contrapone el poder creciente de las autoridades sanitarias y gerencias. Esta situación se puede definir como una Arena Política que conlleva un conflicto permanente pero de intensidad moderada, pero posibilita la supervivencia de la organización, no exento de momentos de fuerte confrontación. En síntesis el colectivo médico ha visto amenazada en parte su autonomía y capacidad decisoria por las normas y controles que las autoridades sanitarias introducen a fin de mantener los resultados de los centros asistenciales dentro de los límites fijados por el poder político.

\section{La oferta sanitaria: Recursos y capacidades}

Finalmente, en este apartado se efectúa una descripción e interpretación de la oferta asistencial utilizando conceptos de la teoría de Recursos y Capacidades. La oferta de medios asistenciales puede entenderse como el conjunto de activos de diferente naturaleza que posibilitan la prestación de atención sanitaria. Dichos activos pueden ser clasificados en recursos tangibles y recursos intangibles. Los recursos tangibles se dividen a su vez en recursos físicos (edificios, equipamientos, terrenos, etc.) y recursos financieros. Los recursos intangibles se caracterizan por estar basados en el conocimiento (información cuya validez ha sido probada) y pueden ser divididos en recursos tecnológicos, reputación y recursos humanos. La tecnología en sentido amplio incluyendo el Know how es un aspecto clave en el desarrollo de la medicina moderna; el dinamismo de las innovaciones en este campo obliga a disponer de capacidad de absorción y flexibilidad para incorporar los continuos avances que se producen. La reputación es un activo intangible que se genera a medida que los clientes de una organización reciben los servicios de la misma y experimentan un conocimiento positivo con los mismos. La reputación de las instituciones sanitarias puede ser entendida como capital confianza, y su importancia es determinante en la viabilidad de las mismas. Finalmente, los recursos humanos, o dicho de forma más precisa el capital humano hace referencia a los conocimientos y habilidades de los profesionales de la sanidad (educación recibida, experiencia acumulada, habilidades poseídas). El potencial de conocimientos a nivel individual es una condición necesaria pero no suficiente para asegurar la contribución de dichos recursos a los 
fines de las organizaciones en que prestan sus servicios. En este sentido la motivación y la cultura dominantes juegan un papel decisivo. Cada vez es más importante la capacidad de trabajo en equipo y las actitudes sociales y sicológicas para completar las habilidades profesionales y técnicas.

Una vez identificados y clasificados los recursos de forma aislada se requiere integrar los mismos para llevar a cabo las diferentes actividades que justifican la existencia de las instituciones asistenciales. Las capacidades o competencias de una organización hace referencia a la integración de diferentes recursos mediante pautas organizativas para llevar a cabo actividades productivas. Así el trabajo de un cirujano no puede realizarse sin un anestesista, personal de enfermería, instrumental quirúrgico, tecnología de imagen, banco de sangre y otros recursos.

Las organizaciones a medida que aumentan su complejidad se caracterizan por la existencia de una arquitectura de capacidades o competencias, que van desde las más sencillas a las más complejas. Así la capacidad de realizar transplantes a nivel nacional requiere la coordinación de un amplio conjunto de recursos o capacidades de orden inferior, mediante pautas organizativas preestablecidas. Similarmente la capacidad de un hospital para atender enfermedades del corazón depende de la integración de capacidades pertenecientes a diagnóstico de pacientes, cirugía cardiovascular, cuidados antes y después de la operación, y servicios administrativos de diferente naturaleza.

Resulta claro que las capacidades necesarias para integrar los servicios asistenciales de diferente naturaleza como la red de hospitales, los servicios de urgencia, la red de atención primaria, y todas ellas entre sí exigen niveles de coordinación y pautas organizativas de orden superior.

De lo dicho se desprende que la gestión de las organizaciones sanitarias es básicamente la gestión de conocimientos en un entorno cambiante, y que si bien el capital humano es el recurso básico, cada vez son más importantes las capacidades organizativas que aseguren la adecuada coordinación de diferentes activos para garantizar una medicina coste efectiva. 
BIBLIOGRAFÍA

GARCíA, R. y J. VeNTURA (1994) La sanidad pública en el horizonte del año 2000. Economía y empresa en Asturias. Madrid: Editorial Civitas.

Grossman, G. (1972) "On the Concept of Health Capital and the Demand for Health", Journal of Political Economy, 2 (March-April), pp. 223-255.

MINTZBERG, H. (1978) La estructuración de las organizaciones. Barcelona: Ariel.

ORTÚN, V. (1990) La economía en sanidad y medicina: Instrumentos y limitaciones. Barcelona: EUGE.

ORTUN RUBIO, V. y G. LóPEZ CASASNOVAS (2002) Financiación capitativa, la articulación entre niveles asistenciales y descentralización de las organizaciones sanitarias, Documento de trabajo 3. Fundación BBVA.

Serra Ramoneda, A. (1993) La empresa. Análisis económico. Barcelona: Labor. 